\title{
STEINITZ CLASSES OF CYCLIC EXTENSIONS OF DEGREE $l^{r}$
}

\author{
ROBERT LONG
}

ABSTRACT. Let $k$ be an algebraic number field and $l$ an odd prime. The set of Steinitz classes of tamely ramified cyclic extensions $K / k$ of degree $l^{r}$ is calculated and shown to be a subgroup of the ideal class group of $k$.

Introduction. In this paper $k$ will denote a (finite) algebraic number field with ring of algebraic integers $O_{k}$. For a finite extension $K / k$ the ring $O_{K}$ of integers in $K$ is a module over $O_{k}$. Such modules are classified up to isomorphism by two invariants: the rank and the Steinitz class. Since the ring $O_{K}$ is uniquely determined within $K$ it is reasonable to denote the Steinitz class of $O_{K}$ as an $O_{k}$-module by $C(K, k)$.

Denote by $G$ a cyclic group of odd prime power order $l^{r}$. As $K$ varies over the normal extensions of $k$ with Galois group isomorphic to $G$ the associated Steinitz class $C(K, k)$ varies over a subset of the ideal class group $C(k)$ of $k$; this subset is denoted $R(k, G)$. It is the set of ideal classes of $k$ which can be realized as the Steinitz class of the ring of integers in a normal extension $K / k$ with Galois group $G$. The subset $R_{t}(k, G)$ of $R(k, G)$ consisting of those classes which correspond to tamely ramified extensions is a subgroup of $C(k)$ which will be explicitly described. The reader may find [4] and [6] to be useful introductory references. In reference [4] the problem was studied for the special case $r=1$. The methods of this paper are the same in broad outline. In order to carry them through it is necessary to be able to construct tamely ramified cyclic extensions $K / k$ of degree $l^{r}$.

The paper is divided into three parts. In the first it is shown that $R_{t}(k, G)$ is contained in a certain subgroup of $C(k)$. In the second is given the procedure for constructing tamely ramified cyclic extensions of degree $l^{r}$, which is then applied in the third to prove that the inclusion shown in part one is in fact an equality.

Received by the editors January 18, 1974 and, in revised form, March 25, 1974. AMS (MOS) subject classifications (1970). Primary 12 A50.

Key words and phrases. Steinitz class. 
The entire investigation is founded on a formula for $C(K, k)$ due to Artin [1]. Following [4], [6] this will be used in the form: $C(K, k)$ is the class of the square root of the relative discriminant ideal when $K / k$ is a normal extension of odd degree.

1. The following standard lemma will be useful:

Lemma. Let $K / k$ be an abelian extension of number fields which is tamely ramified at the prime $p$ of $k$. Then $N(p) \equiv 1 \bmod e(\hbar, K / k)$. ( $N$ de. notes the absolute norm; $e$ the degree of ramification in the indicated extension.)

Proof. Let $D$ denote the decomposition group of $f$ for $K / k$ and $K / \beta$ the residue field of $K$ at some prime $\Re$ which divides $\{$. Define $f: D \rightarrow$ $(K / \beta)^{\circ}$ by $f(\sigma)=\sigma(\pi) / \pi \bmod \Re$ where $\pi \in \Re \backslash \Re^{2}$ is a prime element for $\Re$. The function $f$ satisfies the identity $f(\sigma \tau)=f(\sigma) f(\tau)^{\sigma}$; the exponent $\sigma$ on the right means the automorphism of $K / \beta$ over $k / p$ induced from $\sigma$. Let $T$ denote the inertia group of $p$ in $K / k ; \tau \in T$ iff $\tau$ acts trivially on $K / \beta$. Since $D$ is abelian one sees that $f(\tau)^{\sigma}=f(\tau) \forall \tau \in T$. As every automorphism of $K / \mathbb{\beta}$ over $k / h$ is induced by some element of $D$ it follows that $f(T) \subseteq$ $(k / p))^{x}$. The restriction of $f$ to $T$ is a homomorphism so that $|T| \mid(N(p)-1)$; since $e=|T|$, this completes the proof.

One could also observe that the lemma follows from local class field theory. An extension $K / k$ will be called "of type $G$ " if it is normal and its Galois group is isomorphic to $G$.

Proposition. Let $K / k$ be a tamely ramified extension of type $G$ and let $W$ be the subgroup of $C(k)$ generated by $\left\{C(k): p\right.$ prime, $\left.N(\xi) \equiv 1 \bmod l^{r}\right\}$. Then $C(K, k) \in W^{(l-1) / 2}$.

Proof. Let $\delta$ be the relative discriminant ideal so that $C(K, k)=C\left(D^{1 / 2}\right)$ by Artin's theorem. If a prime $f$ of $k$ splits into $g$ primes in $K$ each of degree $f$ and ramification degree $e$, then the contribution of $p$ to $\delta$ is $\delta_{p}=f^{f g(e-1)}$. Suppose $e=l^{t}, t>0$; then $f g=l^{r-t}$. The lemma implies that $N(p) \equiv 1 \bmod l^{t}$; hence $N\left(p^{l^{r-t}}\right) \equiv 1 \bmod l^{r}$. From this it is easy to conclude that $C\left(\mathfrak{p}^{l^{r-t}}\right)$ $\epsilon W$. In fact $W=\left\{C(a): N(a) \equiv 1 \bmod l^{r}\right\}$; for let $W_{0}$ be the indicated set. Then it is obvious from the definition of $W$ that $W \subseteq W_{0}$. Conversely, suppose $N(a) \equiv 1 \bmod l^{r}$. By the generalized "theorem on primes in an arithmetic progression" there is a prime ideal $q$ such that $a q^{-1}$ is a principal ideal with a generator $\alpha \equiv 1 \bmod l^{r}$ (congruence in the ring $O_{k}$ ). It follows that 
$C(a)=C(q)$ and that $N(q) \equiv 1 \bmod l^{r}$, so that $C(a) \in W$.

The proposition should now be clear since for every prime $k \mid b$ we have seen that $C\left(D_{p}\right) \in W^{l-1}$. It follows from the proposition that $R_{t}(k, G) \subseteq$ $W^{(l-1) / 2}$.

2. In order to prove that $R_{t}(k, G)$ and $W^{(l-1) / 2}$ are actually equal it is necessary to be able to construct extensions $K / k$ of type $G$. This will be done by constructing Kummer extensions of the field $F=k(\zeta)$ where $\zeta$ is a primitive $l^{r}$ th root of unity. In the case $r=1$ which was studied in [4] an extension $L / F$ of degree $l$ which is abelian over $k$ contains a unique subfield $K$ which is cyclic over $k$; when $r>1$ the situation is less simple but there proves to be a degree of uniqueness in any case. The construction which will be given does not produce all possible extensions $K / k$ of type $G$ but does give enough for present needs.

In addition to the notation which has already been introduced is the following:

$$
\begin{aligned}
& \zeta: \text { primitive } l^{r} \text { th root of unity, } \\
& F=k(\zeta), \\
& Z=\text { cyclic group generated by } \zeta, \\
& m=[F: k], \\
& H=\mathrm{Gal}(F / k), \\
& \sigma: \text { a fixed generator of } H, \\
& s: \text { defined by } \sigma(\zeta)=\zeta^{s}, \\
& R=\mathbf{Z} / l^{r} \mathbf{Z}, \\
& k^{\cdot}=k \backslash\{0\} \text { as a group under multiplication. }
\end{aligned}
$$

For $a \in F^{\cdot}$ let $a_{i}=\sigma^{i}(a)$ and $a_{i}$ be an $l^{r}$ th root of $a_{i}$ in some algebraic closure of $k$. Let $N(a)=F\left(a_{0}, \cdots, a_{m-1}\right)$. The field $N(a)$ is unambiguously determined by $a$ even though the $a_{i}$ 's are defined only up to a factor $\zeta^{j}$. When only one $a$ is under consideration $N(a)$ will be written $N$. Let $\&=\operatorname{Gal}(N / k)$, $X=\operatorname{Gal}(N / F)$, and $E=\left\{\beta \in N^{\bullet}: \beta^{l^{r}} \in F\right\}$. There is a nondegenerate bilinear function $\langle\rangle:, E / F^{*} \times X \rightarrow Z=$ the cyclic group generated by $\zeta$ in $F^{\cdot}$ defined by $\langle e, \tau\rangle=r(e) / e$.

Let $\sigma_{1}$ be a fixed element of $\&$ whose restriction to $F$ is the fixed $\sigma \in$ $H . E / F^{\cdot}$ is a left module over the group ring $R H$ by the action $\sigma\left(e F^{\circ}\right)=$ $\sigma_{1}(e) F^{*}$ which will be written $\sigma(e) F^{\cdot}$ from now on. $X$ is a right $R H$-module 
by the action $x^{\sigma}=\sigma_{1}^{-1} x \sigma_{1} ; Z$ is a left $R H$-module for the Galois action of $H$ on $F$. With these actions the isomorphism of abelian groups $\phi: E / F^{*} \rightarrow$ $\operatorname{Hom}(X, Z)$ given by $\phi\left(e F^{\circ}\right)=\langle e, \cdot\rangle$ is an $R H$-isomorphism. $H$ acts on $\operatorname{Hom}(X, Z)$ via the left action on $Z$ and the right action on $X$ so that $\left({ }^{\sigma} f\right)(x)$ $=\sigma\left(f\left(x^{\sigma}\right)\right)$. This means that

$$
\langle\sigma(e), x\rangle=\sigma\left(\left\langle e, x^{\sigma}\right\rangle\right)=\left\langle e, x^{\sigma}\right\rangle^{s}
$$

so that $\left\langle e, x^{\sigma}\right\rangle=\langle\sigma(e), x\rangle^{s^{-1}}=\left\langle s^{-1} \sigma e, x\right\rangle$ where $s^{-1}$ is to be understood as (a unit) in $R$. Observe that $E / F^{\circ}$ and $X$ are both cyclic $R H$-modules. (For example $E / F^{\circ}$ is generated over $R H$ by $a_{0} F^{*}$.)

The first task is to identify the maximal abelian extension of $k$ in $N$. The following result is easy to prove and probably well known.

Lemma. Let $0 \rightarrow X \stackrel{i}{\rightarrow} \& \rightarrow H \rightarrow 1$ be an extension of an abelian group $X$ by a cyclic group $H=\langle\sigma\rangle$. Then the commutator subgroup of $\&$ is $i\left(X^{1-\sigma}\right)$.

From now on let $\alpha=\alpha_{0}$ which will be identified with $\alpha_{0} F^{*}$ as an element of $E / F^{\circ}$. Any cyclic extension of $F$ in $N$ has the form $F\left(\left(\Sigma r_{i} \sigma^{i}\right) \alpha\right)$ for some element $\Sigma r_{i} \sigma^{i} \in R H$. In view of the lemma such an extension is abelian over $k$ iff

$$
\left\langle\left(\sum r_{i} \sigma^{i}\right) \alpha, x^{1-\sigma}\right\rangle=1 \quad \forall x \in X .
$$

Since $\left\langle\left(\Sigma r_{i} \sigma^{i}\right) \alpha, x^{1-\sigma}\right\rangle=\left\langle\left(\Sigma r_{i} \sigma^{i}-\Sigma s^{-1} r_{i} \sigma^{i+1}\right) \alpha, x\right\rangle$, this will be the case iff the element $\Sigma\left(r_{i}-s^{-1} r_{i-1}\right) \sigma^{i}$ in $R H$ annihilates $\alpha$ in $E / F^{*}$. (We agree that $r_{-1}=r_{m-1}$.) As this is certainly the case if each $r_{i}=r s^{-i}$ for some $r \in R$, the following is true.

Proposition. Let $\epsilon=\Sigma s^{-i} \sigma^{i} \in R H$. Then for any $\beta \in E, F(\epsilon \beta)$ is an abelian extension of $k$.

Remark. In the case $r=1$ every cyclic extension $L / F$ of degree $l$ which is abelian over $k$ can be obtained by adjoining $(\epsilon a)^{1 / l}$ for some $a \epsilon$ $F^{\circ}$. The proof of this fact uses the semisimplicity of the group ring $R H$ and the fact that $\epsilon$ is a primitive idempotent in $R H$. In the case being considered here $R H$ is not semisimple and $\epsilon$ is not an idempotent; one would expect that in this case there are extensions $L / F$ of type $G$ which are abelian over $k$ but which are not of the form $L=F\left((\epsilon a)^{1 / l^{r}}\right)$ for any $a \in F^{*}$. Here is an example of such an extension: Let $k=Q, l^{r}=9, F=Q(\zeta)$ where $\zeta$ is a primitive 9th root of unity. $L$ will be the field $F\left(\zeta^{1 / 9}\right)$ which is certainly 
abelian over $Q$. In terms of the action of $R H$ on $F^{*} / F^{\cdot} l^{r}$ it must be shown that $\zeta \neq \epsilon a$ in $F^{\cdot} / F^{\cdot} l^{r}$ for any $a$. (1) $O_{F}=\mathrm{Z}[\zeta]$ is a PID, for the relative class number is 1 (cf. Hasse [3]) and the class number of the maximal real subfield $\mathrm{F}^{+}$is also 1 (cf. tables in [2] or the review problem set III in Samuel [7]). (2) It is now easy to see that if $\zeta=\epsilon a$ then $a$ may be taken as a unit in $F$. (3) Every unit in $F$ has the form $\pm \zeta^{i} \eta$ where $\eta$ is a unit in $F^{+}$. (This follows from Satz 23 or 24 in [3].) (4) As 2 is a primitive root mod 9 we may take $\sigma(\zeta)=\zeta^{2}$. Then for any $\eta \in F^{+}, \epsilon \eta=\Pi \sigma^{i}(\eta)^{s^{-i}}, s^{-1}=5 \bmod$ $9, \epsilon(\eta) \in\left(F^{+}\right)^{9}$. (Note. In general if $a$ lies in a proper subfield of $F$, then $\epsilon a$ is at least an $l$ th power in $F$ but not necessarily an $l^{r}$ th power.) It follows that $\zeta=\epsilon a$ with $a \in F^{\cdot}$ iff $\zeta= \pm_{\epsilon} \zeta^{i}$ for some $i$; this is impossible since $\epsilon \zeta \in F^{3}$, and the example is complete.

Return to the situation described above; $E / F^{*}=R H a$. Let $\Sigma=\Sigma_{i} \sigma^{i} \epsilon$ $R H$ and let $X_{0}$ be the kernel of $\Sigma$ acting on $X$. For any $x \in X,\langle\epsilon a, x\rangle=$ $\left\langle\alpha, x^{\Sigma}\right\rangle$ so that $x \in \operatorname{Gal}(N / F(\epsilon \alpha))$ iff $\left\langle\alpha, x^{\Sigma}\right\rangle=1$. Since $x^{\Sigma}$ is invariant under $H, a$ generates $E / F^{\cdot}$ as an $R H$-module, and the pairing $\langle$,$\rangle is non-$ degenerate, it follows that $\operatorname{Gal}(N / F(\epsilon \alpha))=X_{0}$. The preceding proposition expresses the fact that $X^{1-\sigma} \subseteq X_{0}$. Let $L=F(\epsilon \alpha)$; it remains to decide under what circumstances $L$ contains a subfield $K$ which is cyclic of degree $l^{r}$ over $k$. Of course $\mathrm{Gal}(L / k)$ is an extension of $H$ by a cyclic group of order a divisor of $l^{r}$.

Proposition. If $\alpha_{0}, \cdots, \alpha_{m-1}$ are independent over $R$, then $\operatorname{Gal}(L / k)$ $\cong G \times H$.

Proof. In this case $E / F^{\circ}$ is the free $R H$-module with basis $\{\alpha\}$ and one deduces easily that $X \cong R H$ as well. (In fact $X$ is generated as an $R H$ module by the automorphism which sends $\alpha$ to $\zeta a$, the action of this automorphism on other elements of $E$ being determined by the action of $H$ on $X$ and $E / F^{\circ}$.) It follows that $\& \cong X \cdot H, X_{0}=X^{1-\sigma}$, and $X / X_{0} \cong G$, from which the assertion follows.

The hypothesis in the last result can be stated in terms of the $a_{i}$ 's; namely that $a_{0}, \cdots, a_{n-1}$ should be multiplicatively independent modulo $l^{r}$ th powers in $F^{\circ}$.

If this hypothesis is not satisfied it may happen that a field $L=F(\epsilon \alpha)$ is itself cyclic as an extension of $k$. For example, if $F=Q(\zeta)$, where $\zeta$ is a primitive $l^{r}$ th root of unity and $L=F\left((\epsilon \zeta)^{1 / l^{r}}\right)$, this is the case.

From the last proposition one sees that the field $L$ will contain subfields which are cyclic of degree $l^{r}$ over $k$; the final task is to show that 
when $N / F$ is tamely ramified at least one of these extensions is tamely ramified over $k$.

Proposition. Assume $\alpha_{0}, \cdots, \alpha_{n-1}$ are independent over $R$ and as. sume $N / F$ is tamely ramified. Let $K$ be the subfield of $N$ fixed by $X_{0} \cdot H$, then $K / k$ is a tamely ramified extension of type $G$.

Remark. The reader may wonder why the field $L$ was ever mentioned since $K$ is presented as a subfield of $N$. Of course $K \subseteq L$ and it will be useful in the next paragraph to dispense with $N$.

Proof. Suppose $V=\left\langle\sigma^{h}\right\rangle$ is the (first) ramification group of some prime $\mathfrak{l}$ dividing $(l)$ for the extension $F / k$. The ramification group in $N / k$ of any prime $\left\{\mid \mathcal{L}\right.$ will be generated by an element of the form $x \cdot \sigma^{b}$ whose order, say $l^{n}$, is the same as that of $\sigma^{b}$. In terms of the right action of $H$ on $X$ described above the multiplication in $X \cdot H$ is given by $\left(x_{1} \cdot \sigma^{i}\right)\left(x_{2} \cdot \sigma^{j}\right)=$ $x_{1} x_{2}^{\sigma^{-i}} \cdot \sigma^{i+j}$. Hence $1+\sigma^{-b}+\sigma^{-2 h}+\cdots+\sigma^{-\left(l^{n}-1\right) b}$ annihilates $x_{\text {. Since }} X$ is free as a $V$-module it follows that $x \in X^{1-\sigma^{h}} \subseteq X^{1-\sigma}=X_{0}$, and the proposition is proven.

3. The results of $\$ 2$ make it possible to prove the following

Theorem. $R_{t}(k, G)=W^{(l-1) / 2}$. Given $A \in W$ and any ideal $\mathfrak{b}$ in $O_{k}$, there is a tamely ramified extension $K / k$ of type $G$ for which $C(K, k)=A$ and $\left(\mathfrak{D}_{K / k}, \mathfrak{b}\right)=1$.

Proof (compare Theorem (2.6) in [4]). Let $t>3$ be an odd integer such that $A^{t}=A$ and choose positive integers $h_{1}, \cdots, h_{t}$ relatively prime to $l$ so that $\Sigma h_{i}=l t$. Choose primes $p_{1}, \cdots, p_{t}$ relatively prime to $b$ in $F$ each of which splits completely in $F / k$, no two of which are conjugate in $F / k$, and so that each $p_{i} \cap k$ is in the class $A$. Assume also that all the $p_{i}$ 's are in the same ideal class modulo a sufficiently high power of $l$ and let $q_{i}, i=1, \cdots, t$, be nonconjugate prime ideals in the inverse class. Then the ideal

$$
p_{1}^{b} \cdots p_{t}^{b}{ }^{t}\left(q_{1} \cdots q_{t}\right)^{l}
$$

is a principal ideal generated by some $a^{\prime} \in F$ which is congruent to 1 modulo a high power of $l$. To insure that the conjugates of $a^{\prime}$ will be multiplicatively independent modulo $l^{r}$ th powers in $F$ let $a=\rho a^{\prime}$, where $(\rho)$ is a prime ideal not dividing $b$ which splits completely in $F / k$, and $\rho \equiv 1$ modulo a high power of $l$. The conditions satisfied by $a$ insure that the con- 
jugates $\sigma^{i}(a)$ are independent over $R$ (acting exponentially) and that the field $N(a)$ described in $\$ 2$ is tamely ramified over $F$.

(Indeed, if $\mathfrak{l}$ is a prime divisor of $(l)$ in $F$, the condition $a \equiv 1 \bmod a$ high power of $l$ means that $a$ is an $l^{r}$ th power in the -adic completion of $F$, so that $\mathfrak{l}$ splits completely in $N / F$. The expression "a high power of $l$ " means any power $n$ so high that, for each $L(l)$ in $F, a \equiv 1 \bmod \mathfrak{I}^{n}$ implies $a$ is an $l^{r}$ th power in $F_{1}$.) Now let $K$ be the subfield of $N(a)$ described in §2. Then $L=K F$ and $\delta_{L / F}=\delta_{K / k}$ as ideals in $O_{F}$. Let $P_{i}=\Pi_{j} \sigma^{j}\left(p_{i}\right)$, $Q_{i}=\Pi_{j} \sigma^{j}\left(q_{i}\right)$; these are prime ideals in $O_{k}$. It is easy to see that

$$
\delta_{L / F}=\prod_{i} P_{i}^{l^{r}-1} Q_{i}^{l\left(l^{r-1}-1\right)} \cdot(\rho)^{n}
$$

for some $n$. From this it follows that $C(K, k)=A^{(l-1) / 2}$.

4. In general $R(k, G)$ will be a union of cosets of $R_{t}(k, G)$ within the ideal class group $C(k)$. For a given field $k$ it can be described explicitly in terms of the structure of the groups of principal units in the completions $k_{1}$ of $k$ at primes $\mathfrak{l}(l)$. More specifically suppose that $\mathfrak{l}_{1}, \cdots, \mathfrak{l}_{\boldsymbol{g}}$ are the primes of $k$ which divide $(l)$. Suppose that for each $i$ there is given an ideal $l_{i}{ }_{i}$ which is the discriminant of some extension of type $G$ of the local field $k_{i}=$ completion of $k$ at $\mathfrak{L}_{i}$. Then by Grunwald's theorem there is an extension $K_{1} / k$ of type $G$ with discriminant $\Pi_{i} \mathfrak{L}_{i}^{e_{i}} \cdot \mathfrak{b}$ where $(\mathfrak{b},(l))=1$. (This depends on the fact that $l>2$.) From the proof of the proposition in $\$ 1$ it follows that $C(b) \in W^{(l-1) / 2}$. Now suppose $A \in W$. By the theorem there is a tamely ramified extension $K_{2} / k$ of cype $G$ whose discriminant is relatively prime to (l) $\mathrm{b}$ and for which

$$
C\left(K_{2}, k\right)=\left(A \cdot C(\mathfrak{b})^{-1}\right)^{(l-1) / 2} .
$$

The composite $K_{1} K_{2}$ contains a subfield $K$ which is of type $G$ over $k$ and for which $\delta_{K}=\delta_{1} \delta_{2}$. ( $\delta_{i}$ is the discriminant of $K_{i} / k$. One can take for $K$ the field fixed by the "diagonal subgroup" of $\operatorname{Gal}\left(K_{1} K_{2} / k\right)$, cf. Lemma (4.3) in [4].) It follows that

$$
C(K, k)=A^{(l-1) / 2} \cdot \prod_{i} C\left(\zeta_{i}\right)^{e_{i} / 2}
$$

so that $R(k, G)$ is determined by which exponents $e_{i}$ may occur. An example of a field $k$ and a group $G$, elementary abelian of type $(3,3)$, for which $R(k, G)$ is not a group is given in [5]. Of course when $G$ is cyclic of order $l, R(k, G)$ proves to be a group. I do not yet know exactly what happens in the case $G$ cyclic of order $l^{r}$. 


\section{REFERENCES}

1. E. Artin, Questions de base minimale dans la théorie des nombres algébriques, Algèbre et Théorie des Nombres, Colloq. Internat. du Centre National de la Récherche Scientifique, no. 24, Centre National de la Récherche Scientifique, Paris, 1950. MR $13,113$.

2. B. N. Delone and D. K. Faddeev, Theory of irrationalities of the third degree, Trudy Mat. Inst. Steklov. 11 (1940); English transl., Transl. Math. Monographs, vol. 10, Amer. Math. Soc., Providence, R. I., 1964. MR 2, 349; 28 \#3955.

3. H. Hasse, Über die Klassenzahl abelscher Zahlkörper, Akademie-Verlag, Berlin, 1952. MR 14, 141.

4. R. L. Long, Steinitz classes of cyclic extensions of prime degree, J. Reine Angew. Math. 250 (1971), 87-98.

5. - Steinitz classes of relative Galois extensions, Thesis, University of Illinois, 1970.

6. L. R. McCulloh, Cyclic extensions without relative integral bases, Proc. Amer. Math. Soc. 17 (1966), $1191-1194$. MR 37 \# 1353.

7. P. Samuel, Algebraic theory of numbers, Hermann, Paris, 1967; English transl., Houghton Mifflin, Boston, Mass., 1970. MR 35 \#6643; 42 \# 177.

DEPARTMENT OF MATHEMATICS, UNIVERSITY OF FLORIDA, GAINESVILLE, FLORIDA 32611 